\title{
COMBINATORIAL BROADENING MECHANISM OF O-H STRETCHING BANDS IN H-BONDED MOLECULAR CLUSTERS
}

\author{
G. A. Pitsevich, ${ }^{\text {* }}$ I. Yu. Doroshenko, ${ }^{\text {b }}$ V. E. Pogorelov, ${ }^{\mathrm{b}}$ \\ L. G. M. Pettersson, ${ }^{\text {c }}$ V. Sablinskas, ${ }^{\text {d V. V. Sapeshko, }}{ }^{\text {e }}$ \\ and V. Balevicius ${ }^{d}$
}

UDC 535.34;539.194

A new mechanism for combinatorial broadening of donor-OH stretching-vibration absorption bands in molecular clusters with H-bonds is proposed. It enables the experimentally observed increase of the $O$ - $H$ stretching-vibration bandwidth with increasing number of molecules in H-bonded clusters to be explained. Knowledge of the half-width of the OH stretching-vibration absorption band in the dimer and the number of H-bonds in the analyzed cluster is sufficient in the zeroth-order approximation to estimate the $O$-H stretching-absorption bands in clusters containing several molecules. Good agreement between the calculated and published experimental half-widths of the OH stretching-vibration absorption bands in $\mathrm{MeOH}$ and PrOH clusters was obtained using this approach.

Keywords: absorption-band half-width, H-bonding, molecular cluster.

Introduction. Hydrogen bonds (H-bonds) are attractive to research scientists around the globe [1-11] because they play key roles in many physical, chemical, and biochemical processes. Molecules are known to be combined through H-bonds into complexes and clusters that are highly interesting even today [12-14]. H-bond energies are intermediate between covalent bonds and van der Waals interactions. The simplest alcohols can associate into various clusters through H-bonds and can be considered convenient models for studying cooperative effects and structural changes in molecular clusters [15]. Significant research results were achieved using vibrational spectroscopy in combination with matrix isolation [16-19] and cooled jets [20-24], mainly because spectra of isolated clusters at very low temperatures are observed as narrow absorption bands. Furthermore, the probability that they will overlap is very low because such spectra contain almost no hot bands. Therefore, the spectral characteristics of separate clusters of different sizes can be studied. Nevertheless, several bands associated with $\mathrm{O}-\mathrm{H}$ vibrations remain rather broad. Their half-widths reach tens or hundreds of inverse centimeters even under the aforementioned conditions. Therefore, determination of the factors responsible for the formation of such broad bands remains critical.

Understanding of the band-broadening mechanisms in condensed phases is an especially complicated problem [2528] despite the progress in this area [7, 29-37]. Interest in analyzing the band-broadening mechanisms in the gas phase also has not waned [38-40]. Information that is important for constructing a theory can be obtained experimentally during research on small H-bonded clusters that can be separated according to size. Unfortunately, systematic studies of the band half-width as a function of H-bonded cluster size are practically unknown in the literature. Such studies were recently performed for PrOH clusters in Ar matrices [41] where the half-widths of $\mathrm{OH}$ absorption bands increased smoothly with increasing cluster size. An analogous conclusion was drawn [20] during a study of IR and Raman spectra of small MeOH clusters in cooled jets. The results [20] indicated that bands due to vibrations of various symmetries and even bands belonging to different conformers of clusters containing identical numbers of molecules had practically the same half-width. Such experimental results contradict traditional concepts associating large half-widths of donor $\mathrm{OH}$ stretching vibrations $\left(v_{\mathrm{OH}}\right)$ with a high probability of breaking $\mathrm{H}$-bonds. Today, it is generally recognized that the force and strength of $\mathrm{H}$-bonds calculated per bond increase with increasing cluster size $[5,42,43]$. This more likely reduces the probability that they will break. Another dubious hypothesis associated

*To whom correspondence should be addressed.

${ }^{\mathrm{a}}$ Belarusian State University, 4 Nezavisimost' Ave., Minsk, 220050, Belarus; e-mail: pitsevich@bsu.by; ${ }^{\mathrm{b}}$ Taras Shevchenko National University of Kyiv, Kyiv, Ukraine; ' ${ }^{\mathrm{c}}$ AlbaNova University Center, Stockholm University, Stockholm, Sweden; ${ }^{d}$ Vilnius University, Vilnius, Lithuania; ${ }^{e}$ University of Illinois at Chicago, Chicago, USA. Translated from Zhurnal Prikladnoi Spektroskopii, Vol. 83, No. 3, pp. 354-361, May-June, 2016. Original article submitted November 30, 2015. 
large band half-widths with a high probability of transferring vibrational energy from one equivalent elemental oscillator to another. Modern quantum-chemical calculations of IR spectra of alcohol clusters showed that $\mathrm{O}-\mathrm{H}$ vibrations were not localized on individual groups but were collective in nature with practically equivalent contributions to the potential-energy distribution (PED) [20, 23]. Therefore, the search for universal mechanisms capable of explaining the increase of band halfwidth with increasing molecular cluster size is critical.

The goals of the present work were to use combinatorial elements to analyze the mechanisms by which the halfwidths of $\mathrm{O}-\mathrm{H}$ stretching-vibration absorption bands increase with increasing molecular cluster size assuming that the probability of breaking each individual H-bond is identical and practically independent of the cluster size and to compare the band half-widths of enlarging clusters that were predicted by the combinatorial approach with existing experimental data.

Mathematical Model Describing the Increased Broadening of $\mathbf{O}-\mathbf{H}$ Stretching-Vibration Absorption Bands with Increasing Molecular Cluster Size. Several different broadening mechanisms that can contribute simultaneously to the experimentally observed absorption-band half-width are known. The actual contribution of each known broadening mechanism depends on the experimental conditions. We supposed during an analysis of combinatorial broadening factors that the $v_{\mathrm{OH}}$ absorption-band half-width increased with increasing number of molecules in the cluster because the average lifetime of the excited vibrational state $(\tau)$ decreased. Let us assume that donor $\mathrm{OH}$ groups are being discussed. Then, a dimer with a single H-bond would be the smallest molecular cluster under investigation. We assumed during the analysis of the donor$\mathrm{OH}$ excited vibrational state that it could relax by several mechanisms such as natural broadening due to the occurrence of spontaneous transitions into the ground state; redistribution of vibrational energy into low-energy modes within the cluster [44-46]; high anharmonicity of $\mathrm{O}-\mathrm{H}$ stretching vibrations [47-49]; and cleavage of H-bonds. Each of these mechanisms reduced the average lifetime of the excited vibrational state and could be regarded as homogeneous broadening. In turn, this meant that all inhomogeneous absorption-band broadening mechanisms were excluded from the discussion. Furthermore, it was assumed that the contribution of vibration dephasing mechanisms to the band broadening could be neglected. The examination of the set $Z$ of donor $\mathrm{OH}$ groups in a certain cluster as a collection of equivalent oscillators considered that they formed $Z$ normal modes of the cluster due to force interactions. All donor-OH groups in each of them moved with different phase differences. Each donor group was viewed as a channel through which the collective stretching vibration of cluster donor-OH groups could relax through one of the aforementioned mechanisms. Therefore, the increased half-width of $v_{\mathrm{OH}}$ bands with increasing cluster size could be associated with the increased probability that the collective $v_{\mathrm{OH}}$ normal mode would relax because of the increased number of channels through which this could occur.

Let us designate $\tilde{p}_{1}$ as the total relaxation probability of $v_{\mathrm{OH}}$ per unit time per single $\mathrm{OH}$ group; $\tilde{p}_{Z}$, the relaxation probability of a collective $v_{\mathrm{OH}}$ vibration per unit time per cluster containing $Z \mathrm{H}$-bonds. Let us consider that the relaxation probability of a collective vibration in a cluster with several H-bonds is the same over all channels in the zeroth approximation and independent of the cluster size. Let $\tilde{p}_{1}=1 / 100$ for the dimer (which is possible considering the arbitrary choice of unit time) so that $\tilde{p}_{2}$ in the trimer chain must be found. For this, we examined the equivalent mathematical problem of casting dice with 100 faces, each of which was numbered from 1 to 100. If the appearance of a "1" was considered a positive event, then the dimer behavior was modeled by casting one die. The probability of just a single "1" appearing if two dice were thrown simultaneously had to be found for the trimer chain. The probability is known to be determined by the ratio of the number of positive events $(S)$ to their total number $(T)$. The number of positive events was determined as $S=1+1.99+$ $1.99=199$. Here, the first term is the simultaneous appearance of "1" on both dice; the second, appearance of a "1" on the left die multiplied by the 99 numbers (all except the "1") on the right die; the third, appearance of a "1" on the right die multiplied by the 99 possibilities on the left die. The total was 199 possibilities. The total possibilities was $T=100^{2}$. Then, the probability of the desired event $p_{2}=199 / 100^{2}$. Let us examine the instance of three equivalent H-bonds. This could be a cyclic trimer or a tetramer chain. Now, we have three dice analogous to the preceding ones and find the probability that at least one "1" appears. The number of positive events was $S=1+1.99+1.99+1.99+1.99 .99+1.99 .99+1.99 .99=1+$ $3 \cdot 99+3 \cdot 99 \cdot 99=C_{3}^{3} \cdot 1+C_{3}^{2} \cdot 99+C_{3}^{1} \cdot 99 \cdot 99$. Here, the first term is the appearance of a " 1 " on all three dice; the next three te rms, appearance of a " 1 " on two dice; the final three terms, appearance of a " 1 " on one of the dice. As usual, $C_{n}^{m}=n ! / m !(n-m) !$. In the more general instance, $p=m / k$. Now, the die had $k$ faces. As before, a positive event was the appearance of a "1". However, there were now $m$ "1" digits on $k$ faces and $(k-m)$ digits different from unity. For $Z$ H-bonds ( $Z$ dice),

$$
\begin{aligned}
S=C_{Z}^{Z} m^{Z}(k-m)^{0}+C_{Z}^{Z-1} m^{Z-1}(k-m)^{1} & +C_{Z}^{Z-2} m^{Z-2}(k-m)^{2}+\ldots+C_{Z}^{2} m^{2}(k-m)^{Z-2}+C_{Z}^{1} m^{1}(k-m)^{Z-1} \\
& =k^{Z}-C_{Z}^{0} m^{0}(k-m)^{N} .
\end{aligned}
$$




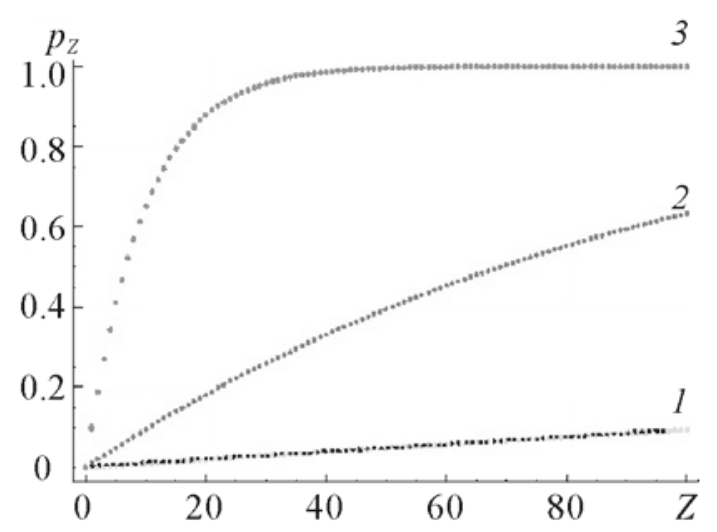

Fig. 1. Dependence of $p_{Z}$ on $Z$ for $p_{1}=0.001$ (1), 0.01 (2), and 0.1 (3).

The total number of possibilities was $k^{2}$. Then, the probability of just a single " 1 " appearing was

$$
p_{Z}=1-C_{Z}^{0} m^{0}(k-m)^{Z} / k^{Z}=1-(k-m)^{Z} / k^{Z}=1-[(k-m) / k]^{Z}=1-(1-m / k)^{Z}=1-\left(1-p_{1}\right)^{Z} .
$$

The form of Eq. (1) indicated that it could be derived by an alternate method. The resulting formula could be analyzed by plotting $p_{Z}$ as a function of $Z$ for various $p_{1}$ values. Figure 1 shows that $p_{Z}$ increased practically linearly with increasing $Z$ for small $p_{1}$ values. However, the function $p_{Z}(Z)$ became nonlinear as $p_{1}$ increased.

Analysis of the Shape and PED of OH Stretching Vibrations in MeOH Clusters. The constructed model assumed that all $\mathrm{O}-\mathrm{H}$ vibrations in clusters with $\mathrm{H}$-bonds were collective. This assumption could be justified theoretically by calculating the shape and PED [50] of normal modes of various MeOH clusters. The calculations were performed in the B3LYP/cc-pVTZ approximation [51-54] using the GAMESS quantum-chemical software [55] for $\mathrm{MeOH}$ clusters containing from three to six molecules. According to the calculations, off-diagonal force constants due to second derivatives of the potential energy over $\mathrm{O}-\mathrm{H}$ valence coordinates of various molecules in the cluster were different from zero. This confirmed that force interaction occurred among $\mathrm{OH}$ vibrations. The fact that $\mathrm{O}-\mathrm{H}$ vibrations were collective could be clearly demonstrated by using calculated contributions to the PED (Table 1) of $\mathrm{O}-\mathrm{H}$ stretching vibrations in the clusters.

Table 1 shows that individual $\mathrm{OH}$ oscillators could be assumed not to contribute to several collective vibrations. However, degenerate (3411.9 and $3412 \mathrm{~cm}^{-1}$ in the tetramer) or quasi-degenerate ( 3575.3 and $3581.4 \mathrm{~cm}^{-1}$ in the trimer) vibrations due to the high symmetries of the corresponding clusters are actually being discussed. In these instances, the PED of both vibrations should more likely be summed. For example, we obtain for the tetramer

$$
50.51 q_{1}^{\mathrm{OH}}+49.7 q_{2}^{\mathrm{OH}}+49.69 q_{3}^{\mathrm{OH}}+50.64 q_{4}^{\mathrm{OH}} .
$$

However, combinatorial broadening of absorption bands in molecular clusters could also be analyzed using an approach where the involvement of each equivalent oscillator was considered in more detail based on the shape and PED of the analyzed collective $\tilde{v}_{\mathrm{OH}}$ vibration.

Establishment of the Function for the Increase of Absorption-Band Half-Width Due to O-H Stretching Vibrations with Increasing Number of Cluster H-Bonds. Because each molecular-cluster channel that is involved in combinatorial absorption-band broadening and the mechanism in general are related to the decreased lifetime of the excited vibrational state $v_{\mathrm{OH}}$, the well-known relationship between the absorption-band half-width $(\Delta v)$ and the average lifetime in the excited vibrational state $(\tau)$ can be used [20, 56-59]

$$
\Delta v \tau=1 / 2 \pi .
$$

It is noteworthy that the probability of deactivating the vibrational state per unit time is [59]

$$
\tilde{p}=d N_{i} / N_{i} d t
$$

The quantity $\tilde{p}$ and the average vibrational state lifetime $\tau$ are related by [59] 
TABLE 1. Potential Energy Distribution for OH Stretching Vibrations in Clusters Containing from Three to Six MeOH Molecules

\begin{tabular}{|c|c|c|}
\hline Cluster & Mode, $\mathrm{cm}^{-1}$ & PED, \% \\
\hline \multirow{3}{*}{ Trimer } & 3509.7 & $31.84 q_{1}^{\mathrm{OH}}+27.57 q_{2}^{\mathrm{OH}}+42.54 q_{3}^{\mathrm{OH}}$ \\
\hline & 3575.3 & $22.05 q_{1}^{\mathrm{OH}}+20.08 q_{2}^{\mathrm{OH}}+58.25 q_{3}^{\mathrm{OH}}$ \\
\hline & 3581.4 & $47.44 q_{1}^{\mathrm{OH}}+52.11 q_{2}^{\mathrm{OH}}$ \\
\hline \multirow{4}{*}{ Tetramer } & 3313.6 & $26.27 q_{1}^{\mathrm{OH}}+24.86 q_{2}^{\mathrm{OH}}+27.2 q_{3}^{\mathrm{OH}}+25.72 q_{3}^{\mathrm{OH}}$ \\
\hline & 3411.9 & $49.7 q_{2}^{\mathrm{OH}}+49.69 q_{3}^{\mathrm{OH}}$ \\
\hline & 3412.0 & $50.51 q_{1}^{\mathrm{OH}}+50.64 q_{4}^{\mathrm{OH}}$ \\
\hline & 3451.7 & $25.11 q_{1}^{\mathrm{OH}}+24.85 q_{2}^{\mathrm{OH}}+25.21 q_{3}^{\mathrm{OH}}+25 q_{4}^{\mathrm{OH}}$ \\
\hline \multirow{5}{*}{ Pentamer } & 3261.3 & $25.21 q_{1}^{\mathrm{OH}}+24.44 q_{2}^{\mathrm{OH}}+25.41 q_{3}^{\mathrm{OH}}+17.3 q_{4}^{\mathrm{OH}}+12.76 q_{5}^{\mathrm{OH}}$ \\
\hline & 3347.0 & $45.69 q_{2}^{\mathrm{OH}}+32.3 q_{3}^{\mathrm{OH}}+18.46 q_{4}^{\mathrm{OH}}$ \\
\hline & 3360.6 & $37.38 q_{1}^{\mathrm{OH}}+8.77 q_{3}^{\mathrm{OH}}+28.32 q_{4}^{\mathrm{OH}}+26.5 q_{5}^{\mathrm{OH}}$ \\
\hline & 3406.9 & $36.89 q_{1}^{\mathrm{OH}}+15.2 q_{2}^{\mathrm{OH}}+33.71 q_{3}^{\mathrm{OH}}+13.97 q_{4}^{\mathrm{OH}}$ \\
\hline & 3420.0 & $14.34 q_{2}^{\mathrm{OH}}+23.9 q_{4}^{\mathrm{OH}}+59.39 q_{5}^{\mathrm{OH}}$ \\
\hline \multirow{6}{*}{ Hexamer } & 3246.4 & $17.75 q_{1}^{\mathrm{OH}}+17.51 q_{2}^{\mathrm{OH}}+19.85 q_{3}^{\mathrm{OH}}+17.84 q_{4}^{\mathrm{OH}}+15.83 q_{5}^{\mathrm{OH}}+16.2 q_{6}^{\mathrm{OH}}$ \\
\hline & 3324.6 & $18.98 q_{1}^{\mathrm{OH}}+31.45 q_{3}^{\mathrm{OH}}+16.32 q_{4}^{\mathrm{OH}}+29.5 q_{6}^{\mathrm{OH}}$ \\
\hline & 3327.7 & $30.56 q_{1}^{\mathrm{OH}}+16.18 q_{2}^{\mathrm{OH}}+17.3 q_{4}^{\mathrm{OH}}+31.88 q_{5}^{\mathrm{OH}}$ \\
\hline & 3387.8 & $24.82 q_{2}^{\mathrm{OH}}+23.6 q_{3}^{\mathrm{OH}}+27.26 q_{4}^{\mathrm{OH}}+24.58 q_{6}^{\mathrm{OH}}$ \\
\hline & 3390.3 & $36.06 q_{1}^{\mathrm{OH}}+8.05 q_{2}^{\mathrm{OH}}+9.91 q_{3}^{\mathrm{OH}}+6.33 q_{4}^{\mathrm{OH}}+32.27 q_{5}^{\mathrm{OH}}+7.99 q_{6}^{\mathrm{OH}}$ \\
\hline & 3410.4 & $16.05 q_{1}^{\mathrm{OH}}+15.79 q_{2}^{\mathrm{OH}}+14.4 q_{3}^{\mathrm{OH}}+16.68 q_{4}^{\mathrm{OH}}+19.55 q_{5}^{\mathrm{OH}}+17.67 q_{6}^{\mathrm{OH}}$ \\
\hline
\end{tabular}

Note. $q_{1}^{\mathrm{OH}}, \ldots, q_{Z}^{\mathrm{OH}}$ are $\mathrm{OH}$ stretching coordinates in a cyclic cluster containing $Z \mathrm{MeOH}$ molecules.

$$
\tilde{p} \tau=1 .
$$

Let us switch to the dimensionless average lifetime in Eq. (4)

$$
\tilde{\tau}=\tau / t_{0} .
$$

By substituting Eq. (5) into Eq. (4), we obtain

$$
\tilde{p} t_{0} \tilde{\tau}=1 .
$$

In order that the dimensionless quantity $\tilde{p} t_{0}$ coincided with the probabilities of a "1" appearing on the dice, i.e., in order that

$$
\tilde{p} t_{0}=p
$$


$t_{0}$ had to be set equal to the $\mathrm{O}-\mathrm{H}$ stretching-vibration period in the $\mathrm{MeOH}$ dimer. In fact, in this instance, $\tilde{\tau}$ turned out to be equal to $\tilde{n}$, the average number of $\mathrm{OH}$ vibrations in the excited state and, according to Eq. (6), only in this instance would the certain event $(p=1)$ correspond as expected to $\bar{n}=1$. Because the wavenumber of the $\mathrm{OH}$ stretching vibration in the $\mathrm{MeOH}$ dimer was $3575 \mathrm{~cm}^{-1}$ [21-24], its period was $T=1 / c \tilde{v} \Rightarrow T \approx 10^{14} \mathrm{~s}$. Thus, $t_{0}=10^{-14} \mathrm{~s}$. Then, we obtain from Eqs. (6) and (7)

$$
p \tilde{\tau}=1 .
$$

Let us switch to wavenumbers in Eq. (2)

$$
\Delta \tilde{v}=\Delta v / c \Rightarrow \Delta v \tau=\Delta \tilde{v} c \tau=1 / 2 \pi \Rightarrow 2 \pi c \Delta \tilde{v} \tau=1
$$

Also, let us switch to the dimensionless average lifetime in Eq. (9)

$$
2 \pi c \Delta \tilde{v} \tau=2 \pi c \Delta \tilde{v} T \tau / T=2 \pi c \Delta \tilde{v} T \tilde{\tau}
$$

Then,

$$
2 \pi c T \Delta \tilde{v} \tilde{\tau}=1 .
$$

By comparing Eqs. (11) and (8), we obtain

$$
p=2 \pi c T \Delta \tilde{v}=2 \pi \lambda \Delta \tilde{v}=2 \pi \Delta \tilde{v} / \tilde{v}
$$

Here and henceforth, $\Delta \tilde{v}_{Z}$ means the $v_{\mathrm{OH}}$ absorption-band half-width in a cluster containing $Z$ H-bonds. Then, Eq. (12) can be rewritten as

$$
p_{Z}=2 \pi \Delta \tilde{v}_{Z} / \tilde{v}_{Z} \text { or } \Delta \tilde{v}_{Z}=\tilde{v}_{Z} p_{Z} / 2 \pi
$$

By substituting Eq. (1) into Eq. (13), we obtain

$$
\Delta \tilde{v}_{Z}=\tilde{v}_{Z} p_{Z} / 2 \pi=\tilde{v}_{Z}\left(1-\left(1-p_{1}\right)^{Z}\right) / 2 \pi=\tilde{v}_{Z}\left(1-\left(1-2 \pi \Delta \tilde{v}_{1} / \tilde{v}_{1}\right)^{Z}\right) / 2 \pi
$$

Equation (14) enables the half-width of absorption bands due to $\mathrm{O}-\mathrm{H}$ stretching vibrations in clusters containing $Z$ H-bonds $\left(\Delta \tilde{v}_{Z}\right)$ to be calculated from the known absorption-band half-width due to donor OH stretching vibrations in the $\operatorname{dimer}\left(\Delta \tilde{v}_{1}\right)$ and the wavenumber $\left(\tilde{v}_{Z}\right)$ of these bands. However, the number of H-bonds in the cluster can be estimated if the experimental values of $\Delta \tilde{v}_{Z}, \tilde{v}_{Z}, \Delta \tilde{v}_{1}$, and $\tilde{v}_{1}$ are known

$$
Z=\ln \left(1-2 \pi \Delta \tilde{v}_{Z} / \tilde{v}_{Z}\right) / \ln \left(1-2 \pi \Delta \tilde{v}_{1} / \tilde{v}_{1}\right)
$$

Comparison of Calculated and Experimental Half-Widths of Absorption Bands Due to O-H Vibrations in Clusters of Various Sizes. The combinatorial mechanism proposed above was assumed to be the main contributor to the tendency of the half-width of $\mathrm{O}-\mathrm{H}$ stretching-vibration absorption bands to increase with increasing number of molecules in the alcohol clusters although several alternate mechanisms for the absorption-band broadening could appear under the experimental conditions. PrOH clusters in matrix isolation were systematically studied earlier [41]. In particular, half-widths of $v_{\mathrm{OH}}$ bands for variously sized clusters were determined.

Table 2 presents the absorption-band half-widths for the trimer chain $(Z=2)$, the cyclic trimer $(Z=3)$, and the cyclic tetramer $(Z=4)$ that were calculated according to Eq. (13) with $\Delta \tilde{v}_{1}=47 \mathrm{~cm}^{-1}$ for the PrOH dimer. The experimental values for the corresponding absorption bands agreed well with those predicted theoretically and are also presented. The experimental data for $\mathrm{MeOH}$ clusters could be compared using previous results [20] where several bands that were assigned to variously sized clusters were observed. The dimer band had a half-width of $6 \mathrm{~cm}^{-1}$. A band of half-width $14.7 \mathrm{~cm}^{-1}$ with two maxima separated from each other by $4.7 \mathrm{~cm}^{-1}$ was assigned to the cyclic trimer. This band resulted from overlap of two bands of approximately identical intensity but smaller half-width. The half-widths of these bands were modeled as 
TABLE 2. Half-widths of O-H Stretching-Vibration Bands for PrOH and $\mathrm{MeOH}$ Clusters Calculated Using a Combinatorial Broadening Model and Their Comparison with Experimental Values

\begin{tabular}{|c|c|c|c|c|c|c|}
\hline \multirow{2}{*}{$Z$} & \multicolumn{4}{|c|}{$\operatorname{PrOH}$} & \multicolumn{3}{c|}{$\mathrm{MeOH}$} \\
\cline { 2 - 7 } & $\Delta \tilde{v}_{Z}^{\text {calc }}, \mathrm{cm}^{-1}$ & $\Delta \tilde{v}_{Z}^{\exp }, \mathrm{cm}^{-1}[41]$ & $\tilde{v}_{Z}^{\exp }, \mathrm{cm}^{-1}[41]$ & $\Delta \tilde{v}_{Z}^{\exp }, \mathrm{cm}^{-1}$ & $\Delta \tilde{v}_{Z}^{\exp }, \mathrm{cm}^{-1}[20]$ & $\tilde{v}_{Z}^{\exp }, \mathrm{cm}^{-1}[20]$ \\
\hline 1 & 47 & $55(3)$ & 3521 & 5.0 & 6.0 & 3575 \\
2 & 88 & $83(5)$ & 3444 & - & - & - \\
3 & 124 & $118(7)$ & 3376 & 14.4 & 8.5 & 3472 \\
4 & 154 & $145(4)$ & 3264 & 18.2 & 21.0 & 3294 \\
\hline
\end{tabular}

Note. $Z$ is the number of H-bonds.

$8.5 \mathrm{~cm}^{-1}$. The band assigned to the cyclic tetramer $\left(3294 \mathrm{~cm}^{-1}\right)$ had a half-width significantly greater than that of the trimer. Furthermore, it overlapped with neighboring bands. This made it complicated to find its half-width. The half-width of the tetramer $v_{\mathrm{OH}}$ band was determined as follows. The unperturbed low-frequency part of the envelope of the lower frequency band was mirrored along a vertical line passing through its maximum. Then, the intensity of the reflected tail was subtracted pointwise from the intensity of the tetramer band. This formed an intermediate envelope for the analyzed band that was still broadened on the high-frequency side. The final band envelope was obtained by reflecting the low-frequency part of the intermediate contour along a vertical line passing through the band maximum. The half-width of this envelope was $21 \mathrm{~cm}^{-1}$. Table 2 presents the absorption-band half-widths of the cyclic trimer $(Z=3)$ and tetramer $(Z=4)$ using Eq. (13) with $\Delta \tilde{v}_{1}=5 \mathrm{~cm}^{-1}$ for the $\mathrm{MeOH}$ dimer. It can be seen that the combinatorial band-broadening mechanism described satisfactorily the experimentally observed tendency of $\Delta \tilde{v}_{Z}$ to increase with increasing $Z$.

The proposed broadening mechanism was rather universal. However, the accuracy of the calculations for clusters with many molecules depended mainly on the accuracy of half-width $\Delta \tilde{v}_{1}$ of the stretching-vibration absorption band for donor $\mathrm{OH}$ groups in the corresponding dimer.

The $\Delta \tilde{v}_{1}$ value should be obtained under the same experimental conditions required to find $\Delta \tilde{v}_{Z}$ or $Z$. Furthermore, one should be convinced that inhomogeneous broadening can be neglected under these conditions. With this condition, an attempt was made to apply this approach to pure liquid $\mathrm{MeOH}$ in order to estimate how much the $\mathrm{H}$-bonds in the liquid are localized or delocalized. The IR spectrum at $203 \mathrm{~K}$ [60] had an absorption band at $3300 \mathrm{~cm}^{-1}$ of half-width $230 \mathrm{~cm}^{-1}$. Setting $\Delta \tilde{v}_{1}=5 \mathrm{~cm}^{-1}$ (absorption-band half-width of the MeOH dimer in the gas phase at $3575 \mathrm{~cm}^{-1}$ ) [20], we obtained $Z=65$. This indicated that the interaction in the condensed phase was highly delocalized. However, $Z=11-12$ was obtained for the liquid by using $27 \mathrm{~cm}^{-1}$ for the half-width of the absorption band at $3525 \mathrm{~cm}^{-1}$ for the $\mathrm{MeOH}$ dimer in an Ar matrix [60] at temperatures below $30 \mathrm{~K}$. Obviously, the result depended strongly on the $\Delta \tilde{v}_{1}$ value. It was concluded that broadening associated with condensation in the solid phase dominated over broadening associated with the temperature because the absorption-band half-width of $\bar{v}_{\mathrm{OH}}$ of the gas-phase dimer was significantly less than that in an Ar matrix. Assuming that broadening in the condensed phase associated with matrix effects could be used to estimate the half-width of a hypothetical $\mathrm{MeOH}$ dimer in the liquid, it was concluded that clusters in the liquid contained from 10 to 20 molecules. However, this was clearly a rough estimate and referred not to specific structures in the liquid but more likely to the effective range of $\mathrm{H}$-bonds. It would have been very useful to study this phenomenon in more detail using, in particular, previous results [61]. However, this was beyond the scope of the present work.

Conclusions. A new model for a universal homogeneous absorption-band broadening mechanism was proposed. It was based on combinatorial relaxation of donor- $\mathrm{OH}$ collective stretching vibrations. An equation relating absorption-band half-widths of collective vibrations to donor-OH absorption-band half-widths in the dimer and the number of elemental oscillators was obtained by using probability theory techniques for a series of combinatorial problems modeling the increased probability of relaxing collective vibrations with an increasing number of elemental oscillators involved in forming them. Band half-widths calculated using this equation and experimental band half-widths due to $\mathrm{OH}$ stretching vibrations in variously sized $\mathrm{PrOH}$ and $\mathrm{MeOH}$ clusters were compared. The $v_{\mathrm{OH}}$ band half-widths of $\mathrm{MeOH}$ cyclic trimer and tetramer were modeled using experimental data [20]. The calculated and experimental values were in satisfactory agreement. The presented combinatorial broadening mechanism was rather universal and described satisfactorily the tendency of the absorption-band half-width to increase with increasing number of cluster H-bonds. 
Acknowledgments. The work was supported financially by the Swedish Scientific Council under a Joint Research Program (Grant No. 2013-6720).

\section{REFERENCES}

1. B. Moulton and M. J. Zaworotko, Chem. Rev., 101, 1629-1658 (2001).

2. T. Steiner, Angew. Chem., Int. Ed., 41, 48-76 (2002).

3. M. S. Taylor and E. N. Jacobsen, Angew. Chem., Int. Ed., 45, 1520-1543 (2006).

4. A. Nilsson, H. Ogasawara, M. Cavalleri, D. Nordlund, M. Nyberg, P. Wernet, and L. G. M. Pettersson, J. Chem. Phys., 122, 154505 (1-9) (2005).

5. L. Ojamae and K. Hermansson, J. Phys. Chem., 98, 4271-4282 (1994).

6. S. J. Grabovski, Hydrogen Bonding. New Insights, Springer, Dordrecht (2006).

7. D. Hadzi, Theoretical Treatment of Hydrogen Bonding, John Wiley \& Sons Ltd., Chichester (1997).

8. XIX Int. Conf. Horizons in Hydrogen Bond Research, Göttingen, Germany (2011); http://www.hbond.de

9. M. A. Suhm, Adv. Chem. Phys., 142, 1-58 (2009).

10. G. A. Pitsevich, A. E. Malevich, E. N. Kozlovskaya, I. Yu. Doroshenko, V. E. Pogorelov, V. Shablinskas, and V. Balevicius, Spectrochim. Acta, Part A, 145, 384-393 (2001).

11. G. Pitsevich and V. Balevicius, J. Mol. Struct., 1072, 38-44 (2014).

12. M. Nyberg, M. Odelius, A. Nilsson, and L. G. M. Pettersson, J. Chem. Phys., 119, 12577-12585 (2003).

13. S. Coussan, Y. Bouteiller, J. P. Perchard, and W. Q. Zheng, J. Phys. Chem. A, 102, 5789-5799 (1998).

14. G. A. Pitsevich, A. E. Malevich, I. Yu. Doroshenko, E. N. Kozlovskaya, V. E. Pogorelov, V. Shablinskas, and V. Balevicius, Spectrochim. Acta, Part A, 120, 585 (2014).

15. I. Doroshenko, G. Pitsevich, V. Shablinskas, and V. Pogorelov, The Cluster Structure of the Liquid Alcohols, Lambert Academic Publishing, Germany (2012).

16. A. J. Barnes and H. E. Hallam, Trans. Faraday Soc., 66, 1920-1931 (1970).

17. L. Schriver, A. Burneau, and J. P. Perchard, J. Chem. Phys., 77, 4926-4933 (1982).

18. S. Coussan, N. Bakkas, A. Loutellier, J. P. Perchard, and S. Racine, Chem. Phys. Lett., 217, 123-130 (1994).

19. S. Coussan, A. Loutellier, J. P. Perchard, S. Racine, A. Peremans, A. Tadjeddine, and W. Q. Zheng, Chem. Phys., 223, 279-292 (1997).

20. R. W. Larsen, P. Zielko, and M. A. Suhm, J. Chem. Phys., 126, 194307 (1-18) (2007).

21. F. Huisken, A. Kulke, C. Laush, and J. M. Lisy, J. Chem. Phys., 95, 3924-3929 (1991).

22. T. Haber, U. Schmitt, and M. A. Suhm, Phys. Chem. Chem. Phys., 1, 5573-5582 (1999).

23. R. A. Provencal, J. B. Paul, K. Roth, C. Chapo, R. N. Casaes, R. J. Saykally, G. S. Tschumper, and H. F. Schaefer III, J. Chem. Phys., 110, 4258-4267 (1999).

24. Y. J. Hu, H. B. Fu, and E. R. Berstein, J. Chem. Phys., 125, 154306 (1-5) (2006).

25. W. E. Donath, J. Chem. Phys., 41, 626-630 (1964).

26. R. G. Gordon, J. Chem. Phys., 44, 3083-3089 (1966).

27. K. H. Illinger and C. O. Trindle, J. Chem. Phys., 48, 4427-4441 (1968).

28. V. E. Pogorelov, A. I. Lizengevich, I. I. Kondilenko, and G. P. Buyan, Usp. Fiz. Nauk, 127, 683-704 (1979).

29. S. Yang, J. Shao, and J. Cao, J. Chem. Phys., 121, 11250-11272 (2004).

30. B. M. Auer and J. L. Skinner, J. Chem. Phys., 127, 104105 (1-10) (2007).

31. B. M. Auer and J. L. Skinner, J. Chem. Phys., 128, 224511 (1-12) (2008).

32. A. Tokmakoff, B. Sauter, and M. D. Fayer, J. Chem. Phys., 100, $9035-9043$ (1994).

33. A. Tokmakoff and M. D. Fayer, J. Chem. Phys., 103, 2810-2826 (1995).

34. K. Nauta and R. E. Miller, J. Chem. Phys., 111, 3426-3433 (1999).

35. R. Venkatramani and S. Mukamel, J. Chem. Phys., 117, 11089-11101 (2002).

36. T. Jansen, W. Zhuang, and S. Mukamel, J. Chem. Phys., 121, 10577-10598 (2004).

37. P. Blaise and O. Henri-Rousseau, Chem. Phys., 256, 85-106 (2000).

38. A. V. Davis, R. Wester, A. E. Bragg, and D. M. Neumark, J. Chem. Phys., 117, 4282-4292 (2002).

39. A. V. Davis, R. Wester, A. E. Bragg, and D. M. Neumark, J. Chem. Phys., 119, 2020-2031 (2003).

40. K.-T. Lee and J. M. Bowman, J. Chem. Phys., 85, 6225-6227 (1986).

41. V. Balevicius, V. Sablinskas, I. Doroshenko, and V. Pogorelov, Ukr. J. Phys., 56, 855-860 (2011). 
42. S. L. Boyd and R. J. Boyd, J. Chem. Theory Comput., 3, 54-61 (2007).

43. F. C. Hagemeister, C. J. Gruenloh, and T. S. Zwier, J. Phys. Chem. A, 102, 82-94 (1998).

44. M. D. Duca, Spectrochim. Acta, Part A, 60, 2667-2671 (2004).

45. L. K. Iwaki and D. D. Dlott, Chem. Phys. Lett., 321, 419-425 (2000).

46. P. Maksyutenko, O. V. Boyarkin, and T. R. Rizzo, J. Chem. Phys., 126, 044311 (1-6) (2007).

47. H. L. Frisch and G. L. Vidale, J. Chem. Phys., 25, 982-986 (1956).

48. R. S. McDowell, J. Chem. Phys., 34, 1065-1066 (1961).

49. R. M. Mazo, J. Chem. Phys., 36, 1893-1984 (1962).

50. G. Keresztury and G. Jalsovszky, J. Mol. Struct., 10, 304-305 (1971).

51. A. D. Becke, J. Chem. Phys., 98, 5648-5652 (1993).

52. C. Lee, W. Yang, and R. G. Parr, Phys. Rev. B: Condens. Matter Mater. Phys., 37, 785-789 (1988).

53. P. J. Stephens, F. J. Devlin, C. F. Chabalowski, and M. J. Frisch, J. Phys. Chem., 98, 11623-11627 (1994).

54. T. H. Dunning, J. Chem. Phys., 90, 1007-1023 (1989).

55. http://www.msg.ameslab.gov/GAMESS/GAMESS.html

56. K. Fujita, T. Fukuda, K. Fukushi, and M. Kimura, J. Raman Spectrosc., 16, 377-385 (1985).

57. B. R. Friedman and M. Schwartz, J. Raman Spectrosc., 15, 273-276 (1984).

58. V. F. Kalasinsky and T. S. Little, J. Raman Spectrosc., 14, 253-258 (1983).

59. M. A. El'yashevich, Atomic and Molecular Spectroscopy [in Russian], Editorial URSS, Moscow (2001).

60. Y. Chernolevskaya, Y. Vaskivskyi, V. Pogorelov, L. G. M. Pettersson, I. Doroshenko, V. Sablinskas, V. Balevicius, J. Ceponkus, A. Malevich, and G. Pitsevich, J. Mol. Liq., 216, 53-58 (2016).

61. P. Kumar, K. T. Wikfeldt, D. Schlesinger, L. G. M. Pettersson, and H. E. Stanley, Sci. Rep., 3, 1980 (1-7) (2013). 